\title{
Beyond the Narrow Resonance Approximation: Decay Constant and Width of the First Pion-Excitation State
}

\author{
Victor Elias and Amir H. Fariborz \\ Department of Applied Mathematics, University of Western Ontario \\ London, Ontario N6A 5B7 Canada \\ Mark A. Samuel * \\ Department of Physics \\ McGill University \\ Montréal, Quebec H3A 2T8 Canada \\ Fang Shi and T. G. Steele \\ Department of Physics and Engineering Physics \\ University of Saskatchewan \\ Saskatoon, Saskatchewan S7N 5C6 Canada
}

(June 23, 1997)

\begin{abstract}
We consider the first pion excitation as a sub-continuum resonance in the pseudoscalar channel, and we obtain parameters characterizing this resonance through a global fit of the Borel-parameter dependence of the field-theoretical pseudoscalar Laplace sum rule to its hadronic (pion + pion-excitation + QCDcontinuum) content. Our analysis incorporates finite-width deviations from the narrow resonance approximation, instanton effects, and higher-loop perturbative contributions to the pseudoscalar correlator. We obtain the following values (uncertainties reflect $90 \%$ confidence levels): mass $\mathrm{M}_{\Pi}=1.15 \pm$ $0.28 \mathrm{GeV}$, width $\Gamma_{\Pi}=<0.48 \mathrm{GeV}$, decay constant $\mathrm{r} \equiv\left[\mathrm{F}_{\Pi} \mathrm{M}_{\Pi}^{2} / \mathrm{f}_{\pi} \mathrm{m}_{\pi}^{2}\right]^{2}=$ $4.7 \pm 2.8$.
\end{abstract}

*Permanent address: Department of Physics, Oklahoma State University, Stillwater, Oklahoma 74078, USA 
The mass, width, and decay constant of the first pion excitation are of interest to effective theories of low energy QCD. Theoretical arguments have been advanced to motivate masses below $1 \mathrm{GeV}$ for this state, ${ }^{1,2}$ despite the $\mathrm{M}_{\Pi}=1300 \pm 100 \mathrm{MeV}$ entry in the present edition of the Particle Data Guide. ${ }^{3}$ The possibility that this resonance may be very broad is also not excluded by present data, which gives a width $\Gamma_{\Pi}$ between 200 and $600 \mathrm{MeV} .{ }^{3} \mathrm{~A}$ broad width is suggestive of a small value for this resonance's decay constant $F_{\Pi}$, which is experimentally undetermined. Recent theoretical work, however, suggests that $F_{\Pi}$ may be very small, even less than $1 \mathrm{MeV}$, if $\mathrm{M}_{\Pi}$ is as large as $1300 \mathrm{MeV} .^{2}$ If the first pion excitation is as light as $1 \mathrm{GeV}$, one cannot automatically assume that this resonance's contribution to the correlation function of pseudoscalar currents is absorbed in the continuum of hadronic states approximated by purely-perturbative QCD, ${ }^{4}$ a continuum whose onset is generally assumed to be substantially above $\mathrm{s}=1 \mathrm{GeV}^{2} .{ }^{5}$ Consequently, a QCD sum-rule approach ${ }^{6}$ to the $\mathrm{I}=1$ pseudoscalar resonances cannot necessarily be limited to the lowest-lying resonance (the pion).

The idea of applying QCD sum-rule methods to the properties of the first pion excitation is an old one. Hubschmid and Mallik ${ }^{7}$ included the first pion-excitation state within a finiteenergy sum-rule analysis of the pseudoscalar channel, assuming a mass $\mathrm{M}_{\Pi}=1.5 \mathrm{GeV}$ based upon a linear Regge trajectory with a universal slope. Their results are suggestive (up to large uncertainties) of a substantially larger value for $\mathrm{F}_{\Pi}$ than anticipated from phenomenological hadronic physics. Their results, however, are obtained within the context of a narrow resonance approximation in which $\Gamma_{\Pi}$ is assumed to be zero, as their analysis was directed more towards a determination of quark masses than of parameters characterizing the first pion excitation.

The work presented here extracts information about the first pion excitation's mass, decay constant, and width from a global fit of the Borel transform of the pseudoscalarcurrent correlator to its hadronic content, assuming that both the pion and the first pion excitation reside below the continuum threshold. Subsequent pion excitations in our analysis are assumed either to be above the continuum threshold, or to have decay constants sufficiently small to decouple them from sub- continuum physics. We depart from prior analyses ${ }^{6,7,8,9,10,11}$ of the pseudoscalar channel sum rules by incorporating 1 ) direct instanton contributions, 2) finite-width effects, and 3) higher-order perturbative contributions to the pseudoscalar current correlation function:

1. Direct Instanton Contributions: In the QCD sum-rule approach, long distance effects are characterized by local matrix elements of quark and gluon operators averaged over the physical vacuum - the QCD-vacuum condensates. However, such local condensates, corresponding to vacuum fluctuations of infinite correlation length, are insufficient to account for the full nonperturbative content of the QCD vacuum, as they do not take into account vacuum fluctuations of non-local origin arising from instantons. ${ }^{12}$ Such finite-correlationlength fluctuations may provide the underlying mechanism for chiral symmetry breaking, ${ }^{13}$ and need to be separately included in an analysis of either scalar or pseudoscalar current correlation functions. ${ }^{10}$ The direct single-instanton contribution to the Borel transform of the pseudoscalar correlation function has been calculated in the instanton liquid model: ${ }^{10,12}$

$$
R_{1}^{\text {inst }}(\tau) \equiv 4 m_{q}^{2} \Pi^{i n s t}(\tau)=\frac{3 m_{q}^{2} \rho_{c}^{2}}{2 \pi^{2} \tau^{3}} e^{-\rho_{c}^{2} / 2 \tau}\left[K_{0}\left(\rho_{c}^{2} / 2 \tau\right)+K_{1}\left(\rho_{c}^{2} / 2 \tau\right)\right],
$$


with instanton size denoted by $\rho_{c}$, and the light quark mass in the $\mathrm{SU}(2)$ - symmetry limit denoted by $\mathrm{m}_{q}$. Note that instanton effects can be "turned off" by letting $\rho_{c} \rightarrow \infty$. In eq. (1), also note that $\Pi(\tau)$ is the Borel transform to the $I=1$ component of the pseudoscalarcurrent correlation function:

$$
\begin{gathered}
\Pi(\tau)=\frac{1}{\pi} \int_{0}^{\infty} \operatorname{Im}\left[\Pi^{p}(s)\right] e^{-s \tau} d s \\
\Pi^{p}\left(q^{2}\right)=i \int d^{4} x e^{i q \cdot x}<\Omega\left|T J^{p}(x) J^{p}(0)\right| \Omega>, \\
J^{p}(x)=\frac{1}{\sqrt{2}}\left[\bar{u}(x) i \gamma_{5} u(x)-\bar{d}(x) i \gamma_{5} d(x)\right] .
\end{gathered}
$$

2. Finite-Width Effects: Conventional methodology for determining the hadronic content of QCD sum-rules rests upon the narrow resonance approximation, in which hadronic BreitWigner contributions to the imaginary parts of appropriate correlators are proportional to $\delta$-functions: 6

$$
\lim _{\Gamma \rightarrow 0} \operatorname{Im}\left[\frac{-1}{s-M^{2}+i M \Gamma}\right]=\pi \delta\left(s-M^{2}\right) .
$$

One can undo the narrow resonance approximation through explicit utilization of the Breit Wigner shape. ${ }^{14}$ Analysis of the longitudinal component of the axial-vector current correlator [corresponding to $4 \mathrm{~m}_{q}^{2} / \mathrm{s}$ times the pseudoscalar current correlator] yields the following hadronic contribution:

$$
\begin{aligned}
R_{1}^{h a d}(\tau)=f_{\pi}^{2} m_{\pi}^{4} e^{-m_{\pi}^{2} \tau} & +\frac{F_{\Pi}^{2} M_{\Pi}^{2}}{\pi} \int_{0}^{s_{0}} \frac{M_{\Pi} \Gamma_{\Pi}}{\left(s-M_{\Pi}^{2}\right)^{2}+M_{\Pi}^{2} \Gamma_{\Pi}^{2}} s e^{-s \tau} d s \\
& +\frac{4 m_{q}^{2}}{\pi} \int_{s_{0}}^{\infty} \operatorname{Im}\left[\Pi^{p}(s)\right]^{\text {pert }} e^{-s \tau} d s .
\end{aligned}
$$

The parameter $s_{0}$ represents the continuum threshold above which hadronic physics coincides with purely-perturbative QCD. In the present work, we first express the imaginary part of the Breit-Wigner shape occurring in (6) as a Riemann sum of unit-area rectangular pulses $P_{m}\left(s, \Gamma^{\prime}\right)$ :

$$
\begin{gathered}
P_{m}\left(s, \Gamma^{\prime}\right) \equiv\left[\Theta\left(s-m^{2}+m \Gamma^{\prime}\right)-\Theta\left(s-m^{2}-m \Gamma^{\prime}\right)\right] / 2 m \Gamma^{\prime}, \\
\frac{M \Gamma}{\left(s-M^{2}\right)^{2}+M^{2} \Gamma^{2}}=\lim _{n \rightarrow \infty} \frac{2}{n} \sum_{j=1}^{n} \sqrt{\frac{n}{j-f}-1} P_{M}\left(s, \sqrt{\frac{n}{j-f}-1} \Gamma\right), \quad[0 \leq f<1] .
\end{gathered}
$$

A semi-analytic approximation for the integral over the Breit- Wigner shape can be realized by noting (for $s_{0}>M_{\Pi}^{2}+M_{\Pi} \Gamma^{\prime}$ ) that

$$
\int_{0}^{s_{0}} P_{M_{\Pi}}\left(s, \Gamma^{\prime}\right) s e^{-s \tau} d s=M_{\Pi}^{2} e^{-M_{\Pi}^{2} \tau} \Delta\left(M_{\Pi}, \Gamma^{\prime}, \tau\right),
$$




$$
\Delta(M, \Gamma, \tau) \equiv \frac{\sinh (M \Gamma \tau)}{M \Gamma \tau}\left[1+\frac{1}{M^{2} \tau}\right]-\frac{\cosh (M \Gamma \tau)}{M^{2} \tau} \underset{\Gamma \rightarrow 0}{\longrightarrow} 1
$$

In the present analysis, we have utilized a 4-pulse approximation to the right-hand side of (8), choosing $\mathrm{f}=0.7$ in order that the area of the four pulses be equivalent to the total area $(=\pi)$ under the Breit-Wigner curve. [In the $n \rightarrow \infty$ limit, such equivalence is true for any choice of $\mathrm{f}$ between 0 and 1 ]. We then obtain a simple expression for the finite-width correction $\mathrm{W}$ to the narrow-resonance-approximation contribution:

$$
\begin{gathered}
\frac{F_{\Pi}^{2} M_{\Pi}^{2}}{\pi} \int_{0}^{s_{0}} \frac{M_{\Pi} \Gamma_{\Pi}}{\left(s-M_{\Pi}^{2}\right)^{2}+M_{\Pi}^{2} \Gamma_{\Pi}^{2}} s e^{-s \tau} d s=F_{\Pi}^{2} M_{\Pi}^{4} e^{-M_{\Pi}^{2} \tau} W\left[M_{\Pi}, \Gamma_{\Pi}, \tau\right] \\
\begin{aligned}
W[M, \Gamma, \tau] & =0.5589 \Delta(M, 3.5119 \Gamma, \tau)+0.2294 \Delta(M, 1.4412 \Gamma, \tau) \\
& +0.1368 \Delta(M, 0.8597 \Gamma, \tau)+0.0733 \Delta(M, 0.4606 \Gamma, \tau) .
\end{aligned}
\end{gathered}
$$

Note that $W \rightarrow 1$ in the narrow-resonance limit $\left(\Gamma_{\Pi} \rightarrow 0\right)$. The four- pulse approximation we utilize serves to mitigate numerical difficulties that arise from the infinite Breit-Wigner tail, which extends unphysically into both Euclidean $(\mathrm{s}<0)$ and post-continuum $\left(\mathrm{s}_{>}>\mathrm{s}_{0}\right)$ values of $\mathrm{s}$.

3. Higher-Order Perturbative Contributions: Virtually all previous sum- rule treatments of the pseudoscalar channel have made use only of the one-loop expression [or incomplete 2-loop expressions] for the purely perturbative QCD-contribution to the pseudoscalar current correlator. However, the perturbative content of the pseudoscalar current correlation function has been known for some time to three-loop order, with higher-loop contributions accompanied by numerically large coefficients: ${ }^{15}$

$$
\begin{aligned}
\operatorname{Im}\left[\Pi^{p}(s)\right]^{\text {pert }} & =\frac{3 s}{8 \pi}\left\{1+\frac{\alpha_{s}}{\pi}\left[\frac{17}{3}-2 \ln (s \tau)\right]\right. \\
& \left.+\left(\frac{\alpha_{s}}{\pi}\right)^{2}\left[40.684-31.667 \ln (s \tau)-1.417 \pi^{2}+4.251 \ln ^{2}(s \tau)\right]+O\left(\alpha_{s}^{3}\right)\right\} .
\end{aligned}
$$

The renormalization point $\mu=\tau^{-1 / 2}$ is chosen to identify the Borel parameter as the renormalization-group ( $\mathrm{RG}$ ) scale parameter. In the work presented here, we have considered both 2- and 3-loop expressions for $\Pi^{p}(s)$ in determining parameters characterizing the first pion excitation. Computational limitations prevented us from determining $90 \%$ confidence-level bounds on our fitted parameters when using the 3- loop expression-we could obtain numerically efficient Monte Carlo estimates of these confidence levels only for the 2 loop case. We were, however, able to compare explicitly the $\chi^{2}$-minimizing fits obtained when either 2- or 3- loop expressions for $\Pi^{p}(s)$ are utilized. Rows 5 and 6 of Table I demonstrate that the pion-resonance mass, decay constant, and width are shifted very little in going from two to three loops, despite potentially large 3-loop perturbative corrections. It should be noted that the minimum $\chi^{2}$ increases by an order of magnitude when the pion resonance is omitted (leaving only the ground state pion). This indicates that, unlike the case for other channels, the pion resonance is too strong to be absorbed into the continuum. 
Our analysis of pion-resonance properties follows from a weighted least squares fit of the $\tau$-dependence $\left[0.4 \mathrm{GeV}^{-2} \leq \tau \leq 2.5 \mathrm{GeV}^{-2}\right]$ of the hadronic contributions to $\mathrm{R}_{1}(\tau)$ to the corresponding field theoretical expression, as derived from the pseudoscalar current correlation function.

The region of $\tau$ is chosen by placing a 20\% upper bound on the theoretical uncertainty in $R_{1}(\tau)$, based upon a $30 \%$ continuum and a $50 \%$ power-law-uncertainty. The hadronic contribution, which explicitly contains the fitted pion-resonance parameters $\mathrm{M}_{\Pi}, \mathrm{F}_{\Pi}, \Gamma_{\Pi}$ and the continuum threshold $s_{0}$, is obtained via substitution of eqs (10-12) into (6). The fieldtheoretical contribution $R_{1}^{f t}(\tau)\left[=4 m_{q}^{2} \Pi(\tau)\right.$ as defined by eq. (2)] contains direct instanton contributions [eq. (1)], QCD-vacuum condensate contributions, ${ }^{6,8,16}$ and purely-perturbative contributions that can be extracted by substitution of (12) into (2). Since the quantity $R_{1}^{f t}(\tau)$ satisfies a renormalization group equation, ${ }^{17}$ coupling constants $\alpha_{s}$ and quark masses $m_{q}$ appearing outside RG-invariant condensates can be replaced with scale-parameter dependent RG-improved expressions referenced to the $\mu=\tau^{-1 / 2}$ mass scale to the appropriate loop level. The two loop expressions are:

$$
\begin{gathered}
\alpha_{s}(\tau)=\frac{2 \pi}{9 L}\left[1-\frac{32 \ln (L)}{81 L}\right], L \equiv-\frac{1}{2} \ln \left(\tau \Lambda^{2}\right), \\
m(\tau)=\hat{m} L^{-4 / 9}\left[1-\frac{0.1989-0.1756 \ln (L)}{L}\right] \equiv \hat{m} \zeta(\tau) .
\end{gathered}
$$

The parameter $\hat{m}$ is the RG-invariant quark mass, which we include as one of our fitted parameters by comparison of the explicit $\tau$ - dependence of

$$
\begin{gathered}
R_{1}^{h a d}(\tau) / \hat{m}^{2}=a\left[1+r e^{-M_{\pi}^{2} \tau} W\left[M_{\Pi}, \Gamma_{\Pi}, \tau\right]+\frac{4 \zeta^{2}(\tau)}{\pi} \int_{0}^{s_{0}} \operatorname{Im}\left[\Pi^{p}(s)\right]^{p e r t} e^{-s \tau} d s\right] \\
a=f_{\pi}^{2} m_{\pi}^{4} / \hat{m}^{2}, \quad r=\left(F_{\Pi} M_{\Pi}^{2} / f_{\pi} m_{\pi}^{2}\right)^{2} .
\end{gathered}
$$

to the corresponding field-theoretical expression:

$$
\begin{aligned}
R_{1}^{f t}(\tau) / \hat{m}^{2} & =\zeta^{2}(\tau)\left\{\frac { 3 } { 2 \pi ^ { 2 } \tau ^ { 2 } } \left[1+\frac{17 \alpha_{s}(\tau)}{3 \pi}+\frac{26.699 \alpha_{s}^{2}(\tau)}{\pi^{2}}+\left(1-\gamma_{E}\right)\left[-2 \alpha_{s}(\tau) / \pi-31.667 \alpha_{s}^{2}(\tau) / \pi^{2}\right]\right.\right. \\
& \left.+\left(\frac{\pi^{2}}{6}+\gamma_{E}^{2}-2 \gamma_{E}\right)\left(\frac{4.251 \alpha_{s}^{2}(\tau)}{\pi^{2}}\right)\right]-4<m \bar{q} q>\frac{1}{2 \pi}<\alpha_{s} G^{2}>+\frac{448 \pi \tau}{27}<\alpha_{s}(\bar{q} q)^{2}> \\
& \left.+\frac{3 \rho_{c}^{2}}{2 \pi^{2} \tau^{3}} e^{-\rho_{c}^{2} / 2 \tau}\left[K_{0}\left(\rho_{c}^{2} / 2 \tau\right)+K_{1}\left(\rho_{c}^{2} / 2 \tau\right)\right]+O\left(m_{q}\right)\right\}
\end{aligned}
$$

$\mathrm{SU}(2)$ breaking effects are higher order in $\mathrm{m}_{q}$, since their contributions to $\mathrm{R}_{1}$ are in terms proportional to $\left(m_{u}-m_{d}\right)^{2}\left(m_{u}+m_{d}\right)^{2} .8$ The contributions to $\mathrm{R}_{1}$ from the dimension-5 quarkantiquark-gluon condensate and the dimension- 6 triple-gluon condensate are proportional to $m_{q}^{3}{ }^{16}$ and are therefore not included in (17). Our fit is obtained using a standard set of perturbative and nonperturbative RG-(quasi-)invariant QCD parameters: $<m \bar{q} q>=$ 
$-f_{\pi}^{2} m_{\pi}^{2} / 4\left[f_{\pi}=131 \mathrm{MeV}\right],<\alpha_{s} G^{2}>=0.045 \mathrm{GeV}^{4}, \Lambda=0.15 \mathrm{GeV}, \rho_{c}=(600 \mathrm{MeV})^{-1}$. Input assumptions concerning the deviation of the dimension- 6 fermion condensate $<\alpha_{s}(\bar{q} q)^{2}>$ from its standard ${ }^{6}$ value $1.8 \cdot 10^{-4} \mathrm{GeV}^{6} \equiv S_{6}$ are specified in Table I, and are discussed below. Optimal values for the parameters a, $\mathrm{r}, \mathrm{M}_{\Pi}, \Gamma_{\Pi}$, and $s_{0}$ correspond to the fit of the $\tau$ dependence of (15) to (17) that minimizes a $\chi^{2}$ weighted for the previously described $50 \%$ uncertainty for power-law corrections and a 30\% uncertainty for continuum corrections. As mentioned earlier, uncertainties in the fitted parameters are obtained only for the two-loop case [for which the integral in (15) can be easily determined analytically] from a MonteCarlo simulation incorporating the power-law and continuum uncertainties described above, as well as a $15 \%$ variation in the value of $\rho_{c}$, and (in rows 1-4 of Table I), a factor of two vacuum-saturation uncertainty in the value $S_{6}$ for $\left\langle\alpha_{s}(\bar{q} q)^{2}\right\rangle$.

The value $S_{6}$ was originally obtained ${ }^{6}$ through use of vacuum saturation $\left[<(\bar{q} q)^{2}\right\rangle=<$ $\left.\bar{q} q>^{2}\right]$ in conjunction with the value for $\langle m \bar{q} q>$ appropriate for a $5 \mathrm{MeV}$ quark mass. Either a heavier quark mass and/or a direct violation of the vacuum-saturation assumption contribute substantial uncertainty to this quantity, which our Monte-Carlo simulation allows to vary over a factor of 2 . To demonstrate the overall insensitivity of our fitting procedure to the value of $\left\langle\alpha_{s}(\bar{q} q)^{2}>\right.$, we have constrained this condensate to equal exactly the standard value $S_{6}$ in rows 5 and 6 of Table I. Comparison of rows 4 and 5 demonstrates that removal of the Monte-Carlo variation in the magnitude of the dimension- 6 condensate has virtually no effect on our results. The results in rows 5 and 6 , however, are indicative of a substantial violation of vacuum-saturation: the standard parameter value $S_{6}$ is much larger than that anticipated for $\left\langle\alpha_{s}(\bar{q} q)^{2}>\right.$ via vacuum saturation if the quark mass at $\mu=1 \mathrm{GeV}$ is of order 8-9 MeV. Row 7 of the Table corresponds to a fit in which exact vacuum-saturation is imposed on the value of $\left\langle\alpha_{s}(\bar{q} q)^{2}>\right.$, by including the implicit dependence of this quantity on the fitted RG-invariant quark mass $\hat{m}$. Thus, the requirements $\left\langle(\bar{q} q)^{2}>=<\bar{q} q>^{2}\right.$ and $<\bar{q} q>=-f_{\pi}^{2} m_{\pi}^{2} / 4 m_{q}$ are simultaneously upheld. When included in our fitting procedure, these input assumptions lead to a $\mu=1 \mathrm{GeV}$ quark mass much larger than $5 \mathrm{MeV}$, although the upper end of the range obtained for $M_{\Pi}$ remains consistent with the $1200 \mathrm{MeV}$ empirical lower bound. ${ }^{3}$

To conclude, $90 \%$ confidence-level results for the first pion-excitation are summarized in rows 4-7 of Table I. These results incorporate 1) the explicit direct-instanton contribution, 2) explicit finite-width effects, 3) the full two- and three-loop perturbative contribution to the sum rule, and 4) the violation [or explicit preservation (row 7)] of the vacuum saturation assumption for the dimension-6 fermionic condensate. We believe the results of row 4 to be phenomenologically salient, as the fitted range for $M_{\Pi}$ is found to be fully inclusive of the present $1300 \pm 100 \mathrm{MeV}$ empirical range. ${ }^{3}$ The final three rows suggest a somewhat lighter pion-excitation than that indicated in the Particle Data Guide, though a mutually consistent value of $1200 \mathrm{MeV}$ is not ruled out.

Although the results of rows 4-7 vary according to the input assumptions employed, a number of common features are evident. The decay constant $\mathrm{F}_{\Pi}$ [using PDG conventions consistent with $\mathrm{f}_{\pi}=131 \mathrm{MeV}$ ] is seen to be at least $2.8 \mathrm{MeV}$, consistent with the larger values of $F_{\Pi}$ already anticipated by Hubschmid and Mallik. ${ }^{7}$ Although little sensitivity is evident to the fitted value of the width (the upper bounds quoted are $90 \%$ confidence levels), values larger than $480 \mathrm{MeV}$ for $\Gamma_{\Pi}$ appear to be ruled out. The fits we obtain are found (surprisingly) to uphold the narrow resonance aproximation $(\Gamma=0)$. The fitted parameter $a$ 
allows a determination of the RG-invariant quark mass $\hat{m}$, and a subsequent determination via (14) of the $\mathrm{SU}(2)$ - symmetric quark mass at a $\mu=1 \mathrm{GeV}$ momentum scale. This value in row 4 is less than values anticipated in the absence of instanton corrections (rows 1 and 3). A comparison of rows 3 and 4 also demonstrates that direct instanton contributions reduce somewhat the value of $\mathrm{M}_{\Pi}$, but increase $\mathrm{F}_{\Pi}$. Finally, we note the relative insensitivity in Table I to the value of the continuum threshold $s_{0}$. The analysis clearly favours a threshold above $M_{\Pi}^{2}$ for the onset of equivalence between perturbative and hadronic QCD. The viability of larger values for $s_{0}$ suggests that smaller values for the pion-excitation decay constant $F_{\Pi}$ could be most easily accommodated by incorporating additional pion resonances into sub-continuum physics.

\section{ACKNOWLEDGEMENTS}

V.E. is grateful for discussions with A. L. Kataev, V. A. Miransky, and A. I. Vainshtein.

M. A. S. would like to thank the Department of Applied Mathematics of the University of Western Ontario for its kind hospitality. This research has been supported by grants from the Natural Sciences and Engineering Research Council of Canada.

\section{REFERENCES}

1. A. A. Andrianov and V. A. Andrianov, Nucl. Phys. B (Proc. Supp't) 39 B, C, 257 (1995).

2. M. K. Volkov and C. Weiss, 1996 preprint hep-ph/9608347 (unpublished).

3. R. M. Barnett et. al. [Particle Data Group], Phys. Rev. D54, 1 (1996).

4. J. J. Sakurai, Phys. Lett. 46B, 207 (1973).

5. L. J. Reinders, H. Rubinstein, and S. Yazaki, Phys. Rep. 127, 1, (1985).

6. M. A. Shifman, A. I. Vainshtein, and V. I. Zakharov, Nucl. Phys. B147, 385 and 448 (1979).

7. W. Hubschmid and S. Mallik, Nucl. Phys. B193, 368 (1981).

8. C. Becchi, S. Narison, E. de Rafael, and F. J. Yndurain, Zeit. Phys. C8, 335 (1981).

9. V. A. Novikov, M. A. Shifman, A. I. Vainshtein, and V. I. Zakharov, Nucl. Phys. B191, 301 (1981).

10. E. V. Shuryak, Nucl. Phys. B214, 237 (1983).

11. J. Gasser and H. Leutwyler, Phys. Rep. 87, 77 (1982). 
12. A. E. Dorokhov, S. V. Esaibegyan, N. I. Kochelev, and N. G. Stefanis, 1996 preprint hep-th/9601086 (unpublished).

13. R. D. Carlitz and D. B. Creamer, Ann. Phys. 118, 429 (1979); D. I. Diakonov and V. Yu. Petrov, Zh. Eksp. Teor. Fiz. 89, 361 and 751 (1985).

14. C. A. Domínguez, Zeit. Phys. C26, 269 (1984).

15. S. G. Gorishny, A. L. Kataev, and S. A. Larin, Phys. Lett. 135B, 457 (1984).

16. E. Bagán, J. I. LaTorre, and P. Pascual, Z. Phys. C32, 43 (1986).

17. S. Narison and E. de Rafael, Phys. Lett. 103B, 57 (1981). 
Table I

\begin{tabular}{|c|c|c|c|c|c|c|c|c|c|c|c|}
\hline \multicolumn{3}{|c|}{ INPUTS } & \multicolumn{9}{|c|}{$\begin{array}{ll}\text { OUTPUTS } \\
\end{array}$} \\
\hline$\rho_{c}\left(\mathrm{MeV}^{-1}\right)$ & $|\Gamma| l$ & $>^{2}$ & & & $M_{\Pi}(\mathrm{GeV})$ & $r$ & $F_{\Pi}(\mathrm{MeV})$ & $a\left(\mathrm{GeV}^{4}\right)$ & $m_{q}[\mu=1 \mathrm{GeV}]$ & $\Gamma(\mathrm{GeV})$ & $s_{0}$ \\
\hline$\infty$ & \begin{tabular}{l|l|l}
0 & 2 \\
\end{tabular} & & & & $2.34 \pm 0.16$ & $8.9 \pm 3.2$ & $4.3 \pm 1.3$ & $0.049 \pm 0.016$ & $9.1 \pm 1.5 \mathrm{MeV}$ & - & \\
\hline $1 / 600$ & $0 \mid 2$ & & & & $.0 \pm 0.25$ & $5.4 \pm 3.8$ & $6.0 \pm 3.7$ & $0.063 \pm 0.035$ & $8.0 \pm 2.2 \mathrm{MeV}$ & - & \\
\hline$\infty$ & -12 & & & & $.31 \pm 0.25$ & $5.7 \pm 2.8$ & $3.6 \pm 1.6$ & $0.048 \pm 0.014$ & $9.2 \pm 1.3 \mathrm{MeV}$ & $<0.50$ & 2 \\
\hline $1 / 600$ & -12 & & & & $.15 \pm 0.28$ & $4.7 \pm 2.8$ & $4.2 \pm 2.4$ & $0.069 \pm 0.037$ & $7.7 \pm 2.2 \mathrm{MeV}$ & $<0.48$ & \\
\hline $1 / 600$ & -12 & & & & $.07 \pm 0.17$ & $4.6 \pm 1.8$ & $4.8 \pm 1.8$ & $0.052 \pm 0.014$ & $8.9 \pm 1.2 \mathrm{MeV}$ & $<0.48$ & \\
\hline $1 / 600$ & -13 & & & & .05 & 4.36 & 4.86 & 0.0622 & $8.10 \mathrm{MeV}$ & 0 & \\
\hline $1 / 600$ & -12 & $\hat{m})^{2}$ & & & $.95 \pm 0.23$ & $6.3 \pm 3.8$ & $7.2 \pm 4.1$ & $0.036 \pm 0.019$ & $10.6 \pm 2.8 \mathrm{MeV}$ & $<0.30$ & \\
\hline
\end{tabular}

Fit of $\Pi(1300)$ pion-resonance parameters to $90 \%$ confidence levels under various input assumptions:

$\rho_{c}=\infty:$ No direct-instanton contribution.

$\Gamma=0$ : Narrow resonance approximation.

$l=2$ : Full two-loop perturbative contribution.

$l=3$ : Full three-loop perturbative contribution to the sum rule. For this case, only the fit which minimizes $\chi^{2}$ is quoted. Computational limitations precluded the determination of $90 \%$ confidence levels.

$S_{6}: S_{6} \equiv 1.8 \cdot 10^{-4} \mathrm{GeV}^{6}$ (see text), corresponding to exact vacuum saturation when $\hat{m} \cong 5$ $\mathrm{MeV}$. Row 7 corresponds to exact vacuum-saturation of $\left\langle\alpha_{s}(\bar{q} q)^{2}>\right.$ for the fitted values of $\hat{m}, m_{q}$. 